\title{
Quantifying the Information Flow between Ghana Stock Market Index and Its Constituents Using Transfer Entropy
}

\author{
Prince Mensah Osei $\mathbb{D I}^{1}$ and Anokye M. Adam (iD) ${ }^{2}$ \\ ${ }^{1}$ Ghana Technology University College, Faculty of IT and Business, Kumasi, Ghana \\ ${ }^{2}$ Department of Finance, School of Business, University of Cape Coast, Cape Coast, Ghana
}

Correspondence should be addressed to Anokye M. Adam; aadam@ucc.edu.gh

Received 16 July 2020; Accepted 12 August 2020; Published 28 August 2020

Academic Editor: Zhengbiao Peng

Copyright (C 2020 Prince Mensah Osei and Anokye M. Adam. This is an open access article distributed under the Creative Commons Attribution License, which permits unrestricted use, distribution, and reproduction in any medium, provided the original work is properly cited.

\begin{abstract}
We quantify the strength and the directionality of information transfer between the Ghana stock market index and its component stocks as well as observe the same among the individual stocks on the market using transfer entropy. The information flow between the market index and its components and among individual stocks is measured by the effective transfer entropy of the daily logarithm returns generated from the daily market index and stock prices of 32 stocks ranging from $2^{\text {nd }}$ January 2009 to $16^{\text {th }}$ February 2018. We find a bidirectional and unidirectional flow of information between the GSE index and its component stocks, and the stocks dominate the information exchange. Among the individual stocks, SCB is the most active stock in the information exchange as it is the stock that receives the highest amount of information, but the most informative source is EGL (an insurance company) that has the highest net information outflow while the most information sink is PBC that has the highest net information inflow. We further categorize the stocks into 9 stock market sectors and find the insurance sector to be the largest source of information which confirms our earlier findings. Surprisingly, the oil and gas sector is the information sink. Our results confirm the fact that other sectors including oil and gas mitigate their risk exposures through insurance companies and are always expectant of information originating from the insurance sector in relation to regulatory compliance issues. It is our firm conviction that this study would allow stakeholders of the market to make informed buy, sell, or hold decisions.
\end{abstract}

\section{Introduction}

Information flow across markets shows how markets depend on each other. Quantifying such information transfer to identify information from dominant markets is of paramount interest to market agents. Earlier studies that focused on detecting and measuring interactions between markets used parametric models. These models assume a linear relationship between the underlying times series of returns of the markets. The predominantly used concept for detecting the lead-lag relationship between time series was Granger causality that requires vector autoregression (VAR) or error correction model (VECM) framework and imposes restrictive assumptions concerning the underlying dynamics [1]. Mere detection of information flows rather than quantifying them does not provide an adequate basis for decision making and that is what Granger causality approach sought to provide. However, information flow as a measure of dependence between financial markets is nonlinear and information theory provides the avenue for characterizing nonlinear dependencies [2].

A stock market is considered a nonisolated system that interacts and exchanges information with the real economy. Information flows between stock markets, a stock market and its constituents, as well as other financial markets. Pricing individual stocks in a market depends on available information to the entire market and information that is more particular to the stock concerned. Models such as the capital asset pricing model (CAPM) explain stock price movements by incorporating market return. Meanwhile, such a model captures the linear correlation between the market return and the individual stock return. The 
constituents of a stock market are interconnected and correlated, and their interactions as a measure of an important internal force have been widely studied using simple correlation function [3-5] and other correlation-based methods such as Granger causality [6]. However, the correlation function has proven to have flaws which include measuring linear relations, even though real interactions between financial times series are nonlinear. Moreover, it only indicates comovement between two variables and does not tell which variable affects which, thus lacking directional information.

The directionality is however required to discriminate the more influential component between correlated participants. Quantifying the flow of information between a stock market and its constituent stocks would help to determine individual stocks that are susceptible to information that is due to changes in the market and its environment as a whole [7]. To be able to quantify the information flow between a stock market and its constituents, as well as among the individual stocks, and to identify the direction of the information flow to determine the most information dominant component, transfer entropy has been applied $[8,9]$. Studies on stock markets using transfer entropy have focused mainly on developed and emerging markets [8-12]. Little or no studies of such nature have focused on developing markets including Ghana Stock Exchange (GSE) and its constituent stocks. Few studies that have focused attention on GSE examined the efficiency of GSE [13-16] and the volatility spillover effects of other financial markets on GSE [17, 18]. Among these studies, enumerated transfer entropy that is capable of quantifying the strength and the direction of interaction between the stock market index and its constituents or other financial markets at the same time is yet to be applied to study information flow dynamics in GSE.

In this paper, we use the transfer entropy pioneered by Schreiber [19] to quantify the direction and the strength of the information transfer between GSE and its constituent stocks and to determine the component that dominates information in the market. We choose transfer entropy which is a kind of log-likelihood ratio [20] for this study because of its ability to quantify information flow based on probability density function. The transfer entropy is a model-free measure which is designed as a Kullback-Leibler distance of transition probabilities, invariance upon nonlinear transformation and can quantify information transfer without being restricted to linear dynamics. To the best of our knowledge, this approach is the first to be adopted to quantify information transfer dynamics of GSE and would help us to address the following questions. First, does the fluctuation in GSE index provide more information to traders who invest in individual stocks? Second, the GSE index and its constituents, which drives the flow of information? Finding answers to these questions would provide more detailed information to traders, investors, and other stakeholders on GSE for the kind of positions they should take regarding a buy, sell, or hold decision and react appropriately to information transfer dynamics.

Ghana provides investment opportunities for international portfolio managers and other foreign investors who seek to diversify their holdings through emerging and developing markets because of the country's political stability enjoyed over the decades. One of the key factors considered by multinational and transnational institutions is the political architecture of a country in assessing its political risk. Because Ghana has been politically stable over a long period through its democratic political system, it is not farfetched to ensure that investors, especially international investors, to be abreast of the dynamics of information flow on the GSE. We believe that a study such as ours is timely to guide both local and international investors who are interested in investing in stocks listed on the GSE to be aware of the information flow dynamics of the GSE and to be able to take the right positions in the market.

Transfer entropy has been applied in the areas of cellular automata of computer science, social networks, statistics, dynamical systems, and finance. Specifically, many scholars have applied transfer entropy to the analysis of stock markets. Marschinski and Kantz [11] examined the information flow between S\&P 500 index and DAX index and found evidence of nonlinear information flow between both indices and introduced now widely applied effective transfer entropy to remove some of the effects of noise from transfer entropy. Oh et al. [12] investigated the information flows among different sectors of the Korean stock market and measured the amount of information flow and the degree of information flow asymmetry between industry sectors. They found that around the subprime crisis, the insurance sector was the key information source. Similarly, Yue et al. [21] performed a comparative analysis of information transfer between industry sectors of the Chinese and the USA stock markets using transfer entropy to measure the information flow from one sector to another. Their study revealed that the most active sector in information exchange is the nonbank financial sector in the Chinese market and the technology sector in the USA market and came to the conclusion that stock markets are more synchronized or ordered, during periods of turmoil than during periods of stability. In a related study, Yue et al. [22] investigated the information flow networks of Chinese stock market sectors using transfer entropy and adopted the maximum spanning arborescence to extract important information flows and the hierarchical structure of the network. They found that the information source was the composite sector while the nonbanking financial sector was the information sink.

In addition to studies that have focused on grouping individual stocks into sectors and examining the information transfer dynamics between sectors, other studies have focused on examining information transfer between stock market indices and their constituents. Baek et al. [23] used transfer entropy to investigate the strength and direction of information transfer in the US stock market and found that companies related to energy industry such as oil, gas, and electricity influenced the whole market. Kwon and Oh [8] observed the asymmetric information flow between the stock market index and composing stocks using transfer entropy. They found that the amount of information flow from index to stock is larger than from stock to index and concluded that the market index plays a role as a major 
driving force to an individual stock. Behrendt et al. [7], on the other hand, quantified and tested for the information flow between two time series with Shannon transfer entropy and Rényi transfer entropy using the $R$ package RTransferEntropy. They applied their methodology after several simulated processes to ten individual stocks that were constituents of the S\&P 500 index as well as the index itself. Their study revealed the presence of bidirectional information flow, but the information flow from the stocks to the market was higher than the other direction.

Despite the contrasting conclusions drawn on using transfer entropy as a proxy for quantifying the information flow between the stock market index and its constituents, there is no study, to the best of our knowledge, that has focused on quantifying the information transfer between GSE index and its constituent stocks as well as quantifying information exchange among GSE's constituent stocks using transfer entropy. We, therefore, apply transfer entropy as a proxy for detecting information flow dynamics on the GSE, and our results reveal the bidirectional and unidirectional flow of information between the market and its constituents, and we find the insurance sector to dominate the information exchange among the sector of Ghana stock market.

The remainder of this study is organized as follows. Section 2 presents the historical background of the Ghana stock market and its development, Section 3 presents the methodology, and Section 4 presents the data. Discussions of results and conclusions are, respectively, presented in Sections 5 and 6 .

\section{Historical Background of Ghana Stock Exchange and Its Development}

Ghana Stock Exchange (GSE), which serves as a platform for electronic securities trading in Ghana, was incorporated in July 1989 and commenced operations in November 1990. GSE started with three stockbrokers, eleven listings, and one commemorative bond. Its processes were mostly manual, and trading was based on a call-over system that was conducted three times each week to fix the price of each listed security from 1991 to 1999 . The call-over system was later replaced by the continuous trading system from 2000 to 2007, where the three days a week price fixing was expanded to daily price fixing and traders manually posted their orders on whiteboards. Despite GSE's laborious manual processes, it recorded a surge in the capital appreciation of 116 per cent three years after commencing operations and was the sixth best performing emerging stock market in 1993. GSE AllShare Index as the index was called became the best index performing stock market among all emerging markets in 1994, gaining 124.3 per cent in its index level. Subsequently, the gains of the index were mixed, falling below the 100 per cent gains and rising above the 100 per cent gains, and by $31^{\text {st }}$ December 2007 and before the introduction of the automated trading system in 2008, GSE has hit a total market capitalization of 12.4 billion cedis with an index appreciation of 31.84 per cent.

Currently, GSE uses the GSE Automated Trading System (GATS) that was introduced in November 2008 to replace the laborious manual trading system. The GATS was introduced to ensure improved efficiency, increased liquidity, and enhanced GSE's competitiveness to attract investors and issuers. GSE as on $31^{\text {st }}$ December 2019 can boast a total listing of forty (40) companies operating in diverse sectors including manufacturing, brewery, banking, insurance, oil, and telecommunication with a total market capitalization of 56.8 billion cedis with less than two (2) listings per year. Lately, the GSE's performance has been abysmal, recording negative gains in the index for the past three consecutive years to 2019, and this, coupled with less liquidity in the market, has resulted in the loss of investor confidence. Notwithstanding the GSE current challenges, the future looks bright as it always has been, and it will continue to provide the platform for securities trading in Ghana.

\section{Methodology}

3.1. Transfer Entropy. To quantify the strength and directionality of information flow between two interacting systems, transfer entropy which was proposed and used by Schreiber [19] is used. This approach for information flow detection between two systems can distinguish driving and responding elements. Developing the transfer entropy formula based on the premise of Shannon entropy, Shannon [24] defines for a discrete random variable $X$ with probability distribution $p\left(x_{t}\right)$ as the average number of bits required to optimally encode independent draws as

$$
H_{X}=-\sum_{x_{t}} p\left(x_{t}\right) \log _{2} p\left(x_{t}\right) .
$$

This definition of entropy measures uncertainty, which increases with the number of bits needed to optimally encode a sequence of realizations of $X$. Note from the subsequent steps that log represents base two logarithms and this base is chosen to mean that the entropy measurement unit is in bits. To measure the information flow between two time series, Shannon entropy is coupled with the concept of Kullback-Leibler distance [25] under the assumption that the underlying processes are Markov processes. Consider a stationary Markov process $X$ of order $k$; then, the probability of observing $X$ at time $t+1$ in state $x_{t}$ conditional on the $k$ previous observations is $p\left(x_{t+1} \mid x_{t}, \ldots, x_{t-k+1}\right)=p$ $\left(x_{t+1} \mid x_{t}, \ldots, x_{t-k}\right)$. The average number of bits needed to encode the observation in $t+1$ if the previous values are known is defined by

$$
h_{X}(k)=-\sum_{x_{t}} p\left(x_{t+1}, x_{t}^{(k)}\right) \log p\left(x_{t+1} \mid x_{t}^{(k)}\right),
$$

where $x_{t}^{(k)}=\left(x_{t}, \ldots, x_{t-k+1}\right)$. Equation (2) is analogous to another Markov process $Y$. Following Schreiber's approach [19], for a bivariate case, the information flow from process $Y$ to process $X$ is determined by quantifying the deviation from the generalized Markov property $p\left(x_{t+1} \mid x_{t}^{(k)}\right)=p\left(x_{t+1} \mid x_{t}^{(k)}, y_{t}^{(l)}\right)$, where $l$ is the order of the Markov process $Y$. When there is no flow of information from $Y$ to $X$, the transition probabilities of $X$ are not influenced by previous observations of $Y$. Relying on the 
Kullback-Leibler distance to measure the deviation of the bivariate processes, Schreiber [19] defines Shannon transfer entropy as

$$
T_{Y \longrightarrow X}(k, l)=\sum p\left(x_{t+2}, x_{t}^{(k)}, y_{t}^{(l)}\right) \log \frac{p\left(x_{t+1} \mid x_{t}^{(k)}, y_{t}^{(l)}\right)}{p\left(x_{t+1} \mid x_{t}^{(l)}\right)}
$$

where $T_{Y \rightarrow X}$ measures the information flow from process $Y$ to process $X$. $T_{X \rightarrow Y}$ which defines the information flow for process $X$ to process $Y$ can be derived analogously. Because transfer entropy is asymmetric, finding the difference between $T_{Y \rightarrow X}$ and $T_{X \rightarrow Y}$ can reveal the dominant direction of information flow.

The observed time series data required to estimate the transfer entropy in equation (3) are continuous. Meanwhile, the transfer entropy measure is derived for discrete data, and therefore, it is required to partition the data into discretized values. Thus, symbolically encode the data points within a given nonoverlapping partition. Partitioning the data into a high number of bins is a likely option, but due to the greater amount of data required for a high number of partitions, we follow the trajectory of the most dominant symbolic encoding approach and partition our dataset into three bins. Given a time series $y(t)$, the symbolically encoded series $S(t)$ can be obtained by partitioning $y(t)$ as follows:

$$
S(t)=\left\{\begin{array}{lll}
1 & \text { for } & y(t) \leq q_{1}, \\
2 & \text { for } & q_{1}<y(t)<q_{2} \\
3 & \text { for } & y(t) \geq q_{2} .
\end{array}\right.
$$

The choice of the bins should be motivated by the distribution of the data. Since tail observations are of essential interest in financial return data, the binning should be done in a manner that the empirical quantile chosen would lead to selecting left and right tail observations into separate bins. To have the tail observations captured into three separate bins as used in our study, using 5\% and 95\% empirical quantiles of the return data, respectively, as lower and upper bounds for the binning, would result in a symbolic encoding where the first (third) bin will include the extreme negative (positive) returns. Specifically, we partition symbolically encoded series $S(t)$ as follows:

$$
S_{t}=\left\{\begin{array}{lll}
1 & \text { for } & y_{t} \leq q_{[0.05]}^{r}, \\
2 & \text { for } & q_{[0.05]}^{r}<y_{t}<q_{[0.95]}^{r}, \\
3 & \text { for } & y_{t} \geq q_{[0.95]}^{r} .
\end{array}\right.
$$

Having symbolically encoded the return and by making the use of chain rule to write the conditional probabilities as fractions of joint probabilities, the probabilities in equation (3) can be calculated by the relative frequencies of all the possible outcomes.

Determining the block lengths of the orders $k$ and $l$ of the Markov process in equation (3) is a nontrivial task when calculating transfer entropy measures and the choice of the block lengths is influenced by the time series that are being analyzed. Large values of $k$ and $l$ are good enough to capture the information flow between two time series, but these may result in finite sample effects. On the other hand, when $k$ in $T_{Y \rightarrow X}(k, l)$ is small, the information contained in the lagged values of $X$ may be wrongly assumed to emanate from $Y$. Hence, following Dimpfl and Peter [10], an appropriate choice of $k$ in the transfer entropy measure $T_{Y \rightarrow X}(k, l)$ can be obtained by calculating the mutual information between the time series and its series with delay $k$ as

$$
M_{x x^{(k)}}=\sum_{x x^{(k)}} p\left(x, x^{(k)}\right) \log \frac{p\left(x, x^{(k)}\right)}{p(x) p\left(x^{(k)}\right)},
$$

where $x^{(k)}$ is the series $x$ with delay $k$. Therefore, the optimal block length $k$ is the value associated with the first local minimum of equation (5) $[26,27]$. To avoid complexity in results interpretation, the block lengths $k$ and $l$ should be set to the same order [19]. Since our study is focused on a financial market, precisely the stock market, immediate past information or only a day before the current day is significant in determining the dynamics of the current day [28]. Therefore, we set the lag orders $k$ and $l$ to unity.

3.2. Effective Transfer Entropy. Estimating transfer entropy measures requires large data size and because large data are hardly available, the estimates are always biased due to small sample effects [11]. According to Marschinski and Kantz [11], the bias can be corrected by calculating a transfer entropy measure called effective transfer entropy (ETE). The ETE is determined by first calculating the transfer entropy of a randomized series where the elements of the time series are individually drawn randomly so that the dependencies between the series and other series are destroyed (shuffling) while maintaining the probability distribution of the shuffled series and then subtracting the shuffled transfer entropy from the transfer entropy. This can be stated mathematically as

$$
\operatorname{ETE}_{Y \longrightarrow X}(k, l)=T E_{Y \longrightarrow X}(k, l)-T E_{Y_{\text {shuffled }} \longrightarrow X}(k, l),
$$

where $\mathrm{TE}_{Y_{\text {shuffed }} \longrightarrow X}(k, l)$ indicates the transfer entropy with series $Y$ shuffled. The shuffling is done by randomly drawing from the distribution of $Y$ and rearranging them to generate a new time series which indicate that all statistical dependencies between $Y$ and $X$ are destroyed as well as time series dependencies of $Y$. Consequently, $\mathrm{TE}_{Y_{\text {shuffed }} X}(k, l)$ converges to zero with increasing sample size and any nonzero value of $\mathrm{TE}_{Y_{\text {shuffled }} X}(k, l)$ is due to small sample effects. To obtain a consistent estimator, the data are shuffled several times and the average transfer entropy estimate over the simulations is computed and eventually subtracted from the unshuffled transfer entropy estimate to obtain a bias-corrected effective transfer entropy estimate.

\section{Data Description}

Daily closing prices of stocks listed on Ghana Stock Exchange (GSE) together with the Ghana Stock Exchange Composite Index (GSECI) from $2^{\text {nd }}$ January 2009 to $16^{\text {th }}$ February 2018, downloaded from GSE website (https://gse. com.gh), are used for this study. Stocks that are listed on the 
GSE over the entire range of the study period are chosen and these include 32 stocks, constituting $80 \%$ of all the listings on the exchange. The companies listed on the GSE are from different sectors of the economy and these include banking, insurance, manufacturing, oil, brewery, and telecommunication. The GSECI itself is based on the volume-weighted average of all individual stocks, and this implies that the size of stock has an impact on the index. Since transfer entropy estimation requires stationary data, we compute log returns from the price series as follows:

$$
y_{i, t}=\ln \left(p_{i, t}\right)-\ln \left(p_{i, t-1}\right)
$$

where $y_{i, t}$ is the return of stock $i$ at time $t$ and $p_{i, t}$ is the closing price of stock $i$ at time $t$. The companies and their corresponding codes are shown in Table 1.

\section{Discussion of Results}

5.1. Effective Transfer Entropy Measure of the Information Flow between GSECI and Its Constituent Stocks. The strength and the direction of information flow between the GSE index and the constituent stocks proxied by the effective transfer entropy are quantified using equation (3) after shuffling the data to account for small sample effects. Figure 1 reports the effective transfer entropy estimates within 95\% confidence intervals for all the selected stocks. The results indicate the presence of both bidirectional and unidirectional information flow between the GSE index and some of the stocks, but the bidirectional information exchanges between the market and its constituents are not significant at the $5 \%$ level of significance. The results also show that quite a substantial number of the stocks neither send nor receive information from the market, and this confirms GSE being less active over the sample period. Among the stocks that appear to send information to the market, only six of them (MLC, ACI, TOT, BOPP, SCB, and $\mathrm{PBC}$ ) significantly transfer information to the market while four stocks (AYRT, HFC, TBL, and GGBL) on the other hand significantly receive information from the market based on the confidence bounds set at 95\% (5\% level of significance). In all, the stocks dominate the information exchange between the market and the stocks. Thus, information flows more from the stocks to the market than in the reverse direction.

5.2. Effective Transfer Entropy Measure and Degree of Asymmetric Information Flow among the Individual Stocks. The stock market as a dynamic complex system involves interactions between the various market components that constitute its constituent stocks. Observing the interactions between the constituents of the market would reflect either the activeness or inactiveness of the market and how the market participants react to news emanating from the market. As discussed in Section 5.1, the proxy for the strength and direction of the information flow is the transfer entropy. To quantify the degree of asymmetric information flow, we follow $\mathrm{Oh}$ et al. [12] and define the degree of
TABLE 1: Listed companies sampled.

\begin{tabular}{|c|c|}
\hline Code & Company \\
\hline AADs & AngloGold Ashanti Depository Shares \\
\hline AGA & AngloGold Ashanti Limited \\
\hline ALW & Aluworks Ltd \\
\hline AYRT & Ayrton Drug Manufacturing Co Ltd \\
\hline BOPP & Benso Oil Palm Plantation Ltd \\
\hline CAL & CAL Bank Limited \\
\hline CLYD & Clydestone (Ghana) Limited \\
\hline CMLT & Camelot Ghana Ltd \\
\hline СРC & Cocoa Processing Company \\
\hline EGL & Enterprise Group Limited \\
\hline ETI & Ecobank Transnational Inc \\
\hline FML & Fan Milk Limited \\
\hline GCB & Ghana Commercial Bank Limited \\
\hline GGBL & Guinness Ghana Breweries Ltd \\
\hline GOIL & Ghana Oil Company Limited \\
\hline GSR & Golden Star Resources Ltd \\
\hline GWEB & Golden Web Limited \\
\hline MLC & Mechanical Lloyd Company Plc \\
\hline PKL & Pioneer Kitchenware Limited \\
\hline PBC & Produce Buying Company Ltd \\
\hline $\mathrm{PZC}$ & PZ Cussons Ghana Ltd \\
\hline HFC & HFC Bank \\
\hline SCB & Standard Chartered Bank Ghana Ltd \\
\hline SCBP & Standard Chartered Bank Ghana Ltd Preference \\
\hline SIC & SIC Insurance Company Limited \\
\hline SPL & Starwin Products Limited \\
\hline $\mathrm{ACI}$ & African Champion Industries Limited \\
\hline SWL & Sam Wood Ltd \\
\hline TBL & Trust Bank Limited (Gambia) \\
\hline TOT & Total Petroleum Ghana Ltd \\
\hline TRANS & Transactional Solutions Ghana \\
\hline UNIL & Unilever Ghana Limited \\
\hline
\end{tabular}

asymmetric information flow to measure the information effect between two the stocks as

$$
\Delta \mathrm{ETE}_{Y \rightarrow X}=\mathrm{ETE}_{Y \rightarrow X}(k, l)-\mathrm{ETE}_{X \longrightarrow Y}(l, k) .
$$

This implies that $\Delta \mathrm{ETE}_{X \rightarrow Y}=-\mathrm{ETE}_{Y \rightarrow X}$. We present the quantified effective transfer entropy measure and degree of asymmetric information flow among the stocks using heat maps shown in Figure 2. Each cell in Figure 2(a) shows the value of ETE while each cell in Figure 2(b) shows the value of $\triangle$ ETE from stock $Y$ to stock $X$. As evidenced in both plots, the diagonal entries of the two heat maps are all zeros, which means that a stock neither transfers nor receives information onto itself. We notice that no stock roughly has highest ETE values for both inflows and outflows among the stocks, and the degree of asymmetric information flow $\triangle \mathrm{ETE}$ (net information flow) is mixed as no stock dominates the information transfer among the stocks. These observations imply that the GSE was less active during the entire sample period. However, the most active stock in information exchange is $\mathrm{SCB}$, but this stock hardly dominated the information flow among all the stocks it interacted with.

Since a stock market is a dynamic system with interacting components, the components exchange information among each other. To be able to identify the most information source and sink stocks, we compute and compare for 


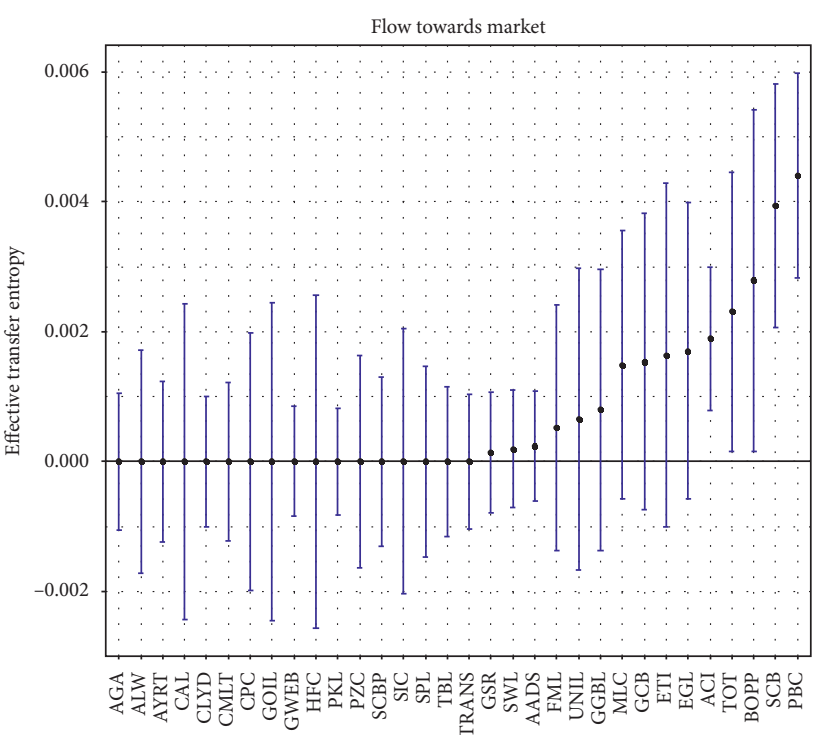

(a)

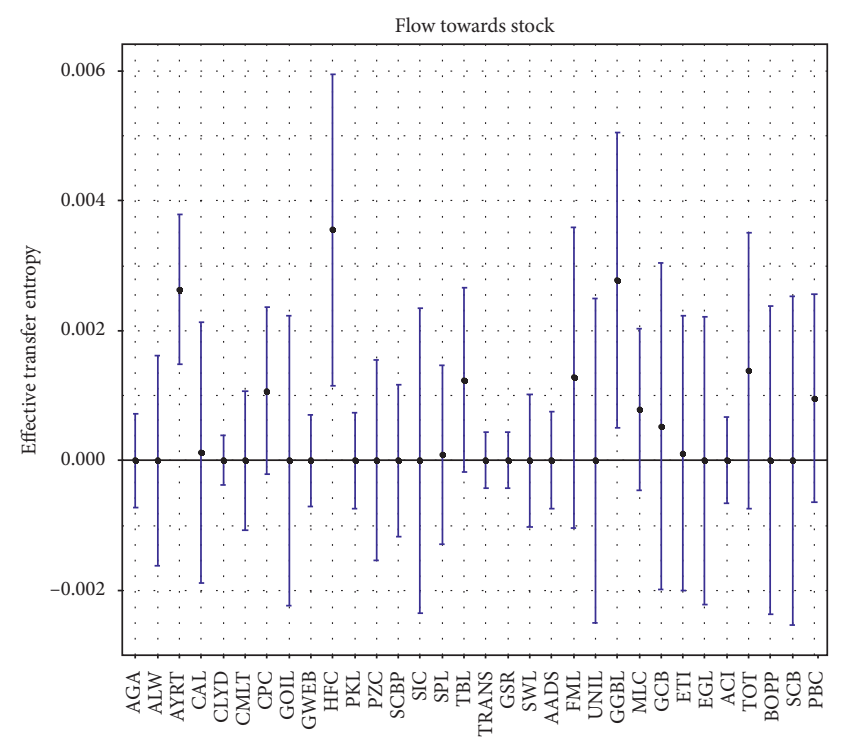

(b)

FIGURE 1: Effective transfer entropy (ETE) estimates together with 95\% confidence bounds for 32 stocks listed on GSE. Both ends of each line segment enclose the ETE within $95 \%$ confidence bounds. A lower end of the line below zero ETE indicates insignificant information flow from either direction at $5 \%$ level of significance.

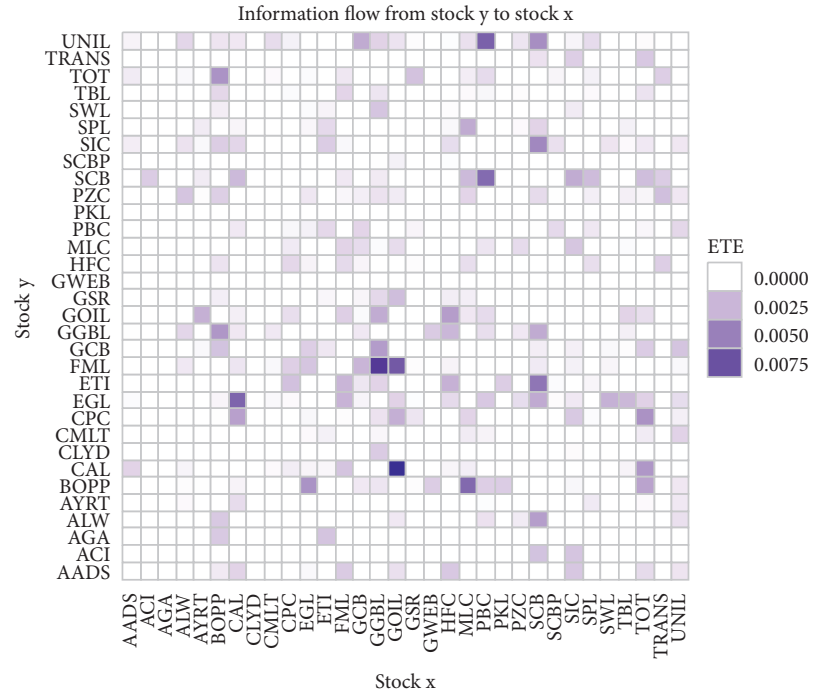

(a)

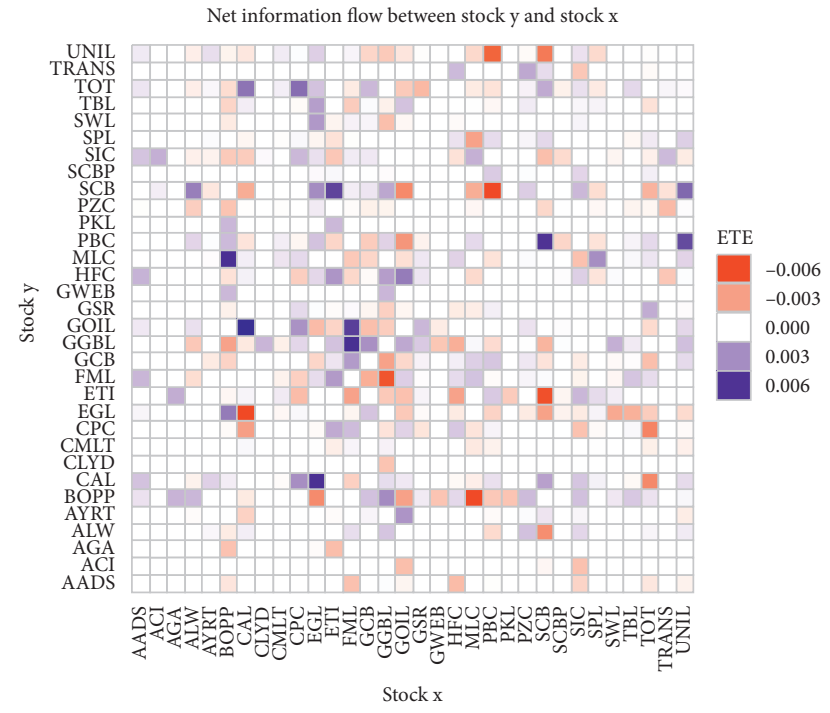

(b)

FIGURE 2: (a) Heat maps of the effective transfer entropy matrix $\mathrm{ETE}_{x, y}$ and (b) the degree of asymmetric information flow $\Delta \mathrm{ETE}_{x, y}$ among the 32 stocks on Ghana stock market (2009-2018).

stock $i$ the average information inflow and outflow following Oh et al. [12] as

$$
\begin{gathered}
F_{\mathrm{in}, i}=\frac{1}{n-1} \sum_{j \neq i} \mathrm{ETE}_{i \longrightarrow j}, \\
F_{\text {out }, i}=\frac{1}{n-1} \sum_{j \neq i} \mathrm{ETE}_{j \longrightarrow i},
\end{gathered}
$$

where $F_{\text {in }, i}$ and $F_{\text {out }, i}$ are, respectively, the average information inflow and outflow of stock $i$. We present the average information inflows and outflows using the bar chart in Figure 3. We notice in the figure that EGL was the most influential stock on GSE during the sample period, followed by FML and UNIL because these stocks send more information to the other stock than receiving from them. On the other hand, the most influenced stock is SCB followed by GOIL and GGBL as these stocks receive more 


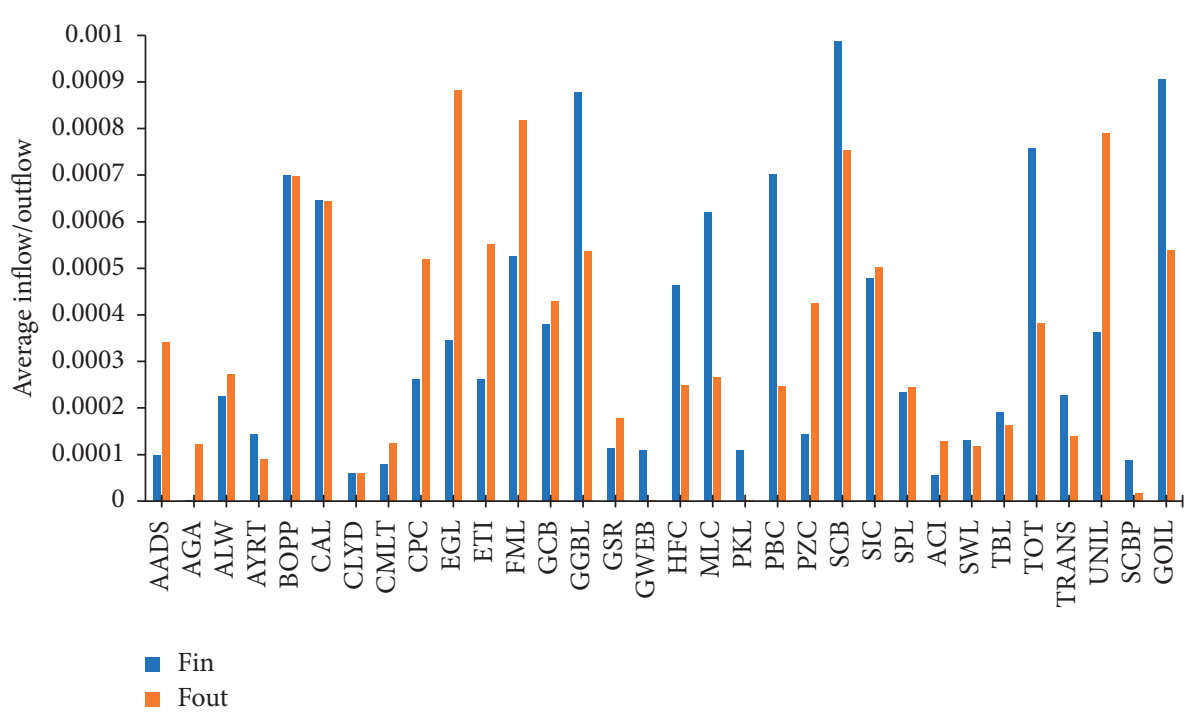

FIgURe 3: Average inflow $F_{\mathrm{in}, i}$ and outflow $F_{\text {out }, i}$ of information for the stocks on Ghana stock market.

information from the other stocks than sending in a reverse manner. Our findings also reveal that CLYD, CAL, and BOPP equally receive and send information from and to other stocks.

We determine the excess of information outflow over the inflow of stock $i$ by calculating the average degree of asymmetric information flow following the approach of $\mathrm{Oh}$ et al. [12] as

$$
\Delta F_{i}=F_{\text {out }, i}-F_{\mathrm{in}, i} .
$$

Figure 4 depicts the average degree of asymmetric information flow $\Delta F$ of the stocks in descending order of net information outflow. We observe highest $\Delta F$ value for EGL and lowest $\Delta F$ value for $\mathrm{PBC}$ which implies that EGL has the highest net outflow of information and thus is the most information dominant stock (big information source) while PBC having the lowest $\Delta F$ (net highest inflow of information) is the most influenced stock (big information sink). In absolute terms, the zero $\Delta F$ values for CLYD, CAL, and BOPP indicate that these stocks equally receive and send information from and to the other stocks. Because in periods of economic turmoil, companies look up to insurance firms for business risk mitigation, the activities of insurance firms are keenly watched by players in the business space quite frequently and based on that, business decisions are made. This is evidenced in our findings as EGL being an insurance company is the most information dominant stock (information source).

\subsection{Determining Degree of Asymmetric Information Transfer} among Stock Market Sectors. We spice up our analysis by determining the most information dominant stock listed on the GSE by categorizing the stocks selected for our study into industry sectors. The stocks listed on GSE are categorized into 8 industry sectors which include basic materials, health care, consumer goods, finance, technology, metal manufacturers, oil and gas, and consumer services. Based on our findings above, we further categorize the financials into banking and insurance to bring the total number of sectorial categorizations to 9 . To facilitate the analysis of our findings, the sectors are assigned with codes, and this is shown in Table 2.

By invoking equations (10) and (11), we calculate the total information flow from sector $j$ to sector $i$ as

$$
\begin{aligned}
& F_{J \longrightarrow I}=\sum_{j \neq i} F_{\mathrm{in}, i}, \\
& F_{I \longrightarrow I}=\sum_{j \neq i} F_{\text {out }, i},
\end{aligned}
$$

where $F_{J \longrightarrow I}$ and $F_{I \longrightarrow J}$, respectively, represent the total amount of information received by sector $i$ and total amount of information sent from sector $i$. We further compute the amount of net information outflow $F$ of sector $i$ (degree of asymmetric information transfer) to determine the most influential sector as

$$
F=F_{\text {out }, i}-F_{\text {in }, i} .
$$

We present in Figure 5 the net outflow of information $F$ of the sectors in descending order. We observe that the insurance sector (code 005) has the highest $F$ value among all the sectors while the $F$ value for oil and gas (code 008) is the lowest. The findings show that the insurance sector has the highest net outflow of information and thus is the most influential sector with regard to information transfer among sectors of the GSE. If we consider the GSE as an information transfer system, the insurance sector is a big information source, influencing other sectors, while the oil and gas sector is a big information sink, influenced by other sectors. Surprisingly, the traditional banking (code 004) and the oil and gas sectors that are supposed to lead the market in terms 


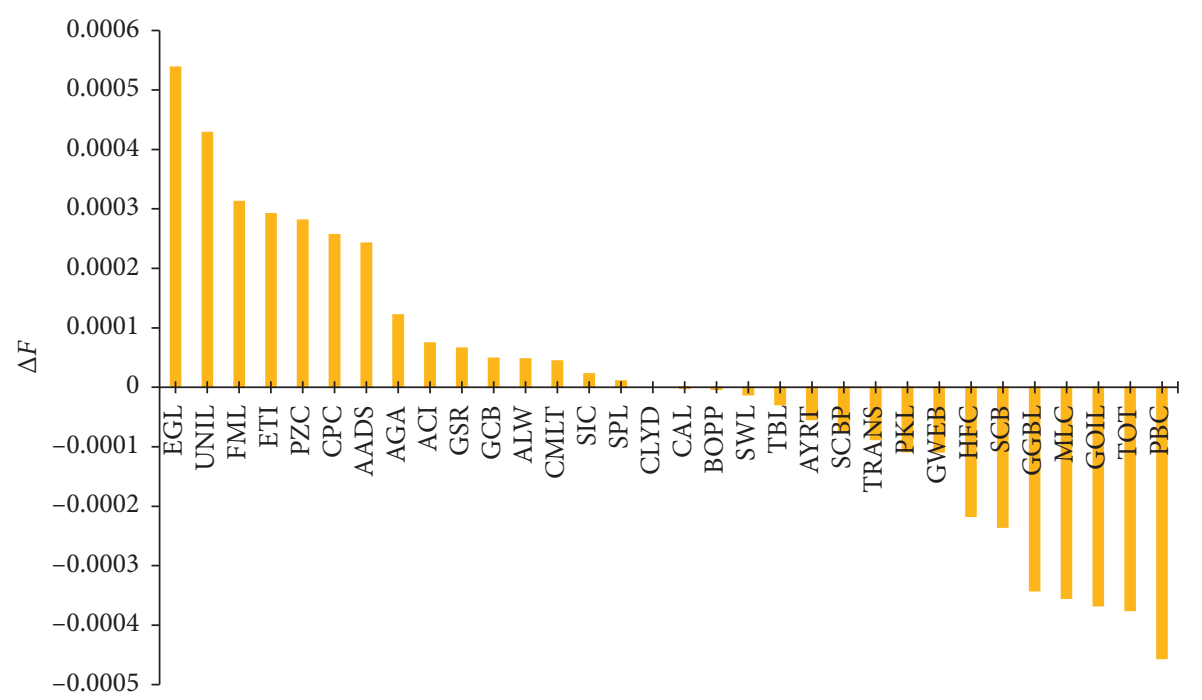

Figure 4: Degree of asymmetric information flow (net information outflow) $\Delta F$ of stocks in descending order.

TABLE 2: Categorizing the stocks into sectors.

\begin{tabular}{lc}
\hline Code & Sector \\
\hline 001 & Basic Materials \\
002 & Health Care \\
003 & Consumer goods \\
004 & Banking \\
005 & Insurance \\
006 & Technology \\
007 & Industrials \\
008 & Oil and gas \\
009 & Consumer services \\
\hline
\end{tabular}

of information flow are rather being influenced by the other sectors. These findings are consistent with the findings of $\mathrm{Oh}$ et al. [12] but differ from the findings of Yue et al. [21] who find the banking sector to be the most influential sector in the China stock market and the energy sector that includes oil and gas to be the most influential sector among the US stock market sectors.

The insurance sector's dominance in the information transfer among the sectors of Ghana stock market is not surprising because of its intermediary role in the financial markets as banks and other businesses rely on insurance companies to mitigate their risk exposures. In addition, insurance companies are important for financial systems' stability because they represent large investors in the financial markets, their growing links with banks, and their function of protecting individuals and companies from different risks (ECB, 2009).

\section{Conclusion}

We calculated the effective transfer entropy and used it as a proxy for quantifying the strength and directionality of information flow between GSE index and its constituents and among 28 listed stocks on the GSE as well. We used daily logarithm returns computed from daily stock prices of the selected stocks and the GSE index, spanning from the period $2^{\text {nd }}$ January 2009 to $16^{\text {th }}$ February 2018. The results reveal interesting findings regarding the information transfer between the GSE index and its constituents and the information exchange among the stocks on GSE. First, we find both bidirectional and unidirectional flow of information between GSE and its constituent stocks. However, the bidirectional information flows are not significant at $5 \%$ level of significance. In all, 6 stocks out of the 32 stocks significantly send information to the market, while 4 out of the 32 stocks significantly receive information from the market. The stocks are more dominant in the information exchange with the market. This revelation indicates how less active and less liquid the GSE has been over the sample period and this serves as a disincentive for investors who would like to invest in stocks on the market.

Second, we observe the information flow dynamics among the individual stocks by calculating effective transfer entropy and asymmetric information flow (net information flow). We find SCB to be the most active stock in information exchange with other stocks. However, concerning the net information flow, EGL is the information source with the highest net outflow of information, while on the other hand, $\mathrm{PBC}$ is the information sink with the highest net inflow of information (least net information outflow). This finding implies that EGL is an insurance company and because all businesses rely on insurance companies for mitigating their risk exposures, companies are interested in news emanating from insurance companies that relate to regulatory compliance issues. To further summarize our findings based on sectors of the GSE, we categorize the stocks into 9 sectors and find the insurance sector (code 005) to be the biggest source of information (highest net 


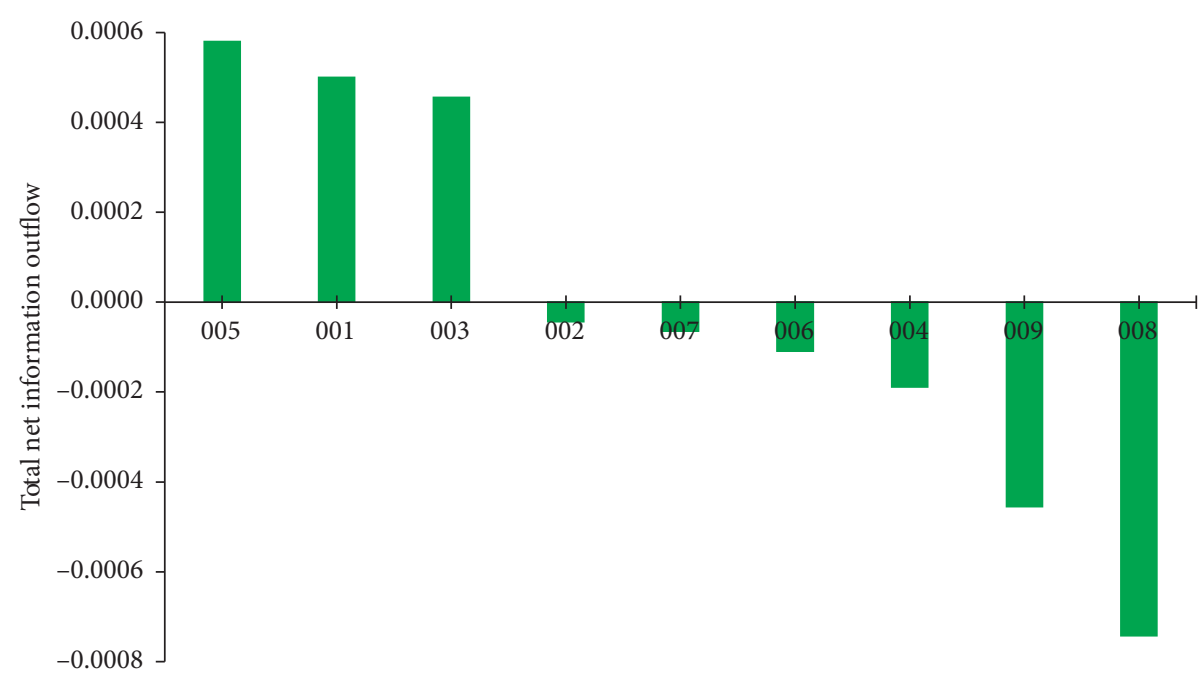

FIGURE 5: Degree of asymmetric information flow $F$ (total net information outflow) of sectors of Ghana stock market in descending order.

information outflow) while the oil and gas sector (code 008 ) is the biggest information sink (highest net information inflow). This result indicates that like any other industry sectors, oil and gas sector having high inherent risks in its operations is always interested in news originating from the insurance sector and the oil and gas sector is highly dependent on insurance sector in periods of disaster.

Our study results provide an avenue for different market players to make informed buy, sell, or hold decisions on the Ghana stock market. Portfolio managers and institutional and individual investors would be able to discriminate between information dominant stocks on the market which would help them to identify and mitigate the risks associated with information-sensitive stocks promptly.

\section{Abbreviations}

AADs: AngloGold Ashanti Depository Shares

AGA: AngloGold Ashanti Limited

ALW: Aluworks Ltd

AYRT: Ayrton Drug Manufacturing Co Ltd

BOPP: Benso Oil Palm Plantation Ltd

CAL: $\quad$ CAL Bank Limited

CLYD: Clydestone (Ghana) Limited

CMLT: Camelot Ghana Ltd

CPC: Cocoa Processing Company

EGL: Enterprise Group Limited

ETI: Ecobank Transnational Inc

FML: $\quad$ Fan Milk Limited

GCB: $\quad$ Ghana Commercial Bank Limited

GGBL: Guinness Ghana Breweries Ltd

GOIL: Ghana Oil Company Limited

GSR: Golden Star Resources Ltd

GWEB: Golden Web Limited

MLC: Mechanical Lloyd Company Plc

PKL: $\quad$ Pioneer Kitchenware Limited

PBC: $\quad$ Produce Buying Company Ltd
PZC: PZ Cussons Ghana Ltd

HFC: HFC Bank

SCB: $\quad$ Standard Chartered Bank Ghana Ltd

SCBP: Standard Chartered Bank Ghana Ltd Preference

SIC: $\quad$ SIC Insurance Company Limited

SPL: $\quad$ Starwin Products Limited

ACI: African Champion Industries Limited

SWL: $\quad$ Sam Wood Ltd

TBL: $\quad$ Trust Bank Limited (Gambia)

TOT: Total Petroleum Ghana Ltd

TRANS: Transactional Solutions Ghana

UNIL: Unilever Ghana Limited

GSE: Ghana Stock Exchange

ETE: Effective transfer entropy.

\section{Data Availability}

The stock indices data used to support the findings of this study were supplied by Ghana Stock Exchange under license and so cannot be made freely available. Requests for access to these data should be made to Ghana Stock Exchange, https://gse.com.gh.

\section{Disclosure}

This study was carried out as part of the employment of first and second authors as lecturers at Ghana Technology University College and University of Cape Coast, respectively.

\section{Conflicts of Interest}

The authors declare that they have no conflicts of interest.

\section{References}

[1] C. W. J. Granger, "Investigating causal relations by econometric models and cross-spectral methods," Econometrica, vol. 37, no. 3, pp. 424-438, 1969. 
[2] H. Haken, Information and Self-Organization, SpringerVerlag, Berlin,Germany, 2006.

[3] L. Kullmann, J. Kert'esz, and K. Kaski, “Time-dependent cross-correlations between different stock returns: a directed network of influence," Physical Review Letters, vol. 66, Article ID 026125, 2002.

[4] R. N. Mantegna, "Hierarchical structure in financial markets," European Physical Journal B, vol. 11, pp. 193-197, 2012.

[5] J. P. Onnela, A. Chakraborti, K. Kaski, J. Kert'esz, and A. Kanto, Physical Review Letters, vol. 68, Article ID 056110, 2003.

[6] J. Shan and N. Pappas, "The relative impacts of Japanese and US interest rates on local interest rates in Australia and Singapore: a granger causality test," Applied Financial Economics, vol. 10, no. 3, pp. 291-298, 2000.

[7] S. Behrendt, T. Dimpfl, F. Peter, and D. Zimmermann, "RTransferEntropy: quantifying information flow between different time series using effective transfer entropy," SoftwareX, vol. 10, p. 100265, 2019, https://CRAN.R-project.org/ package $=$ RtransferEntropy.

[8] O. Kwon and G. Oh, "Asymmetric information flow between market index and individual stocks in several stock markets," EPL (Europhysics Letters), vol. 97, no. 2, Article ID 28007, 2012.

[9] O. Kwon and J. S. Yang, "Information flow between composite stock index and individual stocksflow between composite stock index and individual stocks," Physica A: Statistical Mechanics and Its Applications, vol. 387, no. 12, pp. 2851-2856, 2008.

[10] T. Dimpfl and F. J. Peter, "Using transfer entropy to measure information flows between financial markets," Studies in Nonlinear Dynamics and Econometrics, vol. 17, no. 1, pp. 85-102, 2013.

[11] R. Marschinski and H. Kantz, "Analysing the information flow between financial time series," The European Physical Journal B, vol. 30, no. 2, pp. 275-281, 2002.

[12] G. Oh, T. Oh, H. Kim, and O. Kwon, "An information flow among industry sectors in the Korean stock marketflow among industry sectors in the Korean stock market," Journal of the Korean Physical Society, vol. 65, no. 12, pp. 2140-2146, 2014.

[13] J. M. Frimpong, "Capital market efficiency: an analysis of weak-form efficiency on the Ghana stock exchange," Journal of Money, Investment and Banking, vol. 5, pp. 5-12, 2008.

[14] J. T. Mensah, M. Pomaa-Berko, and K. P. Adom, "Does automation improve stock market efficiency in Ghana," $A f$ rican Review of Economics and Finance, vol. 6, no. 1, pp. 69-101, 2014.

[15] A. K. Osei, "Asset pricing and information efficiency of the Ghana stock market," AERC Research Paper 115, 2002.

[16] G. Tweneboah, A. N. Amanfo, and S. P. Kumah, "Evidence of market inefficiency and exchange rate predictability in Ghana," Ghanaian Journal of Economics, vol. 1, no. 1, pp. 52-66, 2013.

[17] B. Lin, P. K. Wesseh, and M. O. Appiah, "Oil price fluctuation, volatility spillover and the Ghanaian equity market: implication for portfolio management and hedging effectiveness," Energy Economics, vol. 42, pp. 172-182, 2014.

[18] M. M. Zankawah and C. Stewart, "Measuring the volatility spill-over effects of crude oil prices on the exchange rate and stock market in Ghana," The Journal of International Trade \& Economic Development, vol. 29, no. 4, pp. 420-439, 2019.

[19] T. Schreiber, "Measuring information transfer," Physical Review Letters, vol. 85, no. 2, pp. 461-464, 2000.
[20] L. Barnett and T. Bossomaier, "Transfer entropy as a loglikelihood ratio," Physical Review Letters, vol. 109, Article ID 138105, 2012.

[21] P. Yue, Y. Fan, A. J. Batten, and W.-X. Zhou, "Information transfer between stock market sectors: a comparison between the USA and China," Entropy, vol. 22, no. 194, Article ID e22020194, 2020

[22] P. Yue, Q. Cai, W. Yan, and W. X. Zhou, "Information flow networks of Chinese stock market sectorsflow networks of Chinese stock market sectors," IEEE Access, vol. 8, no. 1, pp. 13066-13077, 2020.

[23] S. K. Baek, W. S. Jung, O. Kwon, and H.-T. Moon, "Transfer entropy analysis of the stock market," 2005, http://arxiv.org/ abs/physics/0509014.

[24] C. E. Shannon, "A mathematical theory of communication," Bell System Technical Journal, vol. 27, no. 3, pp. 379-423, 1948.

[25] S. Kullback and R. A. Leibler, "On information and sufficiency," The Annals of Mathematical Statistics, vol. 22, no. 1, pp. 79-86, 1951.

[26] A. M. Fraser and H. L. Swinney, "Independent coordinates for strange attractors from mutual information," Physical Review $A$, vol. 33, no. 2, pp. 1134-1140, 1986.

[27] Y. V. Reddy and A. Sebastin, "Are commodity and stock markets independent of each other? a case study in India," Journal of Alternative Investments, vol. 11, pp. 85-99, 2009.

[28] L. Sandoval, "Structure of a global network of financial companies based on transfer entropyfinancial companies based on transfer entropy," Entropy, vol. 16, no. 8, pp. 4443-4482, 2014.

[29] European Central Bank (ECB), Financial Stability Review: December 2009, European Central Bank (ECB), Frankfurt, Germany, 2009, https://www.ecb.europa.eu/pub/pdf/fsr/ financialstabilityreview200912en.pdf. 